\title{
Modeling and simulation of complex flows using Basset digital filters
}

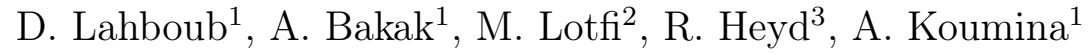 \\ (1) Equipe de Physique des Nanostructures (EPN), Université Cadi Ayyad, ENS Marrakech, Maroc. \\ (2) Laboratoire Matériux, Energie \& Environnement (LaMEE), Université Cadi Ayyad, ENS Marrakech, \\ Maroc. \\ (3) Laboratoire Angevin de Mécanique, Procédés et InnovAtions (LAMPA), ENSAM ParisTech Angers, \\ France.
}

\begin{abstract}
:
Taking into account the Basset force (memory term), in the balance of the forces exerted on a colloidal particle (CP) suspended in a fluid, results in an equation of motion of integrodifferential form. This type of equation allows for example to modeling a colloidal particle settling in an quiescent fluid or in a fluid flowing at low particle's Reynolds number. It also allows to study the transport of pathogens via aerosols, thus giving access to important information on the airborne propagation of respiratory viruses, such as COVID-19 and its variants for example.

Most studies of the PCs motion in a fluid are usually simplified by not taking into account the Basset memory force, as it considerably complicates the numerical solution of the equations of motion of these PCs. This simplification can lead to considerable errors in the evaluation of the trajectory and velocity of the PCs, which can subsequently lead to errors in the calculation of the physical and rheological properties of colloidal suspensions.

The present study deals with the numerical solution of Basset's integro-differential equation, by two significantly different approaches, namely : a piecewise linear approximation (PLA) and the method of Basset numerical filters (BNF). These methods are first exposed and compared on test cases, they are then applied to the study of the sedimentation of spherical PCs with micrometer radii. This study has shown that the usual dynamics of PCs, which does not take into account the Basset memory term, can be very different from the exact dynamics using the Basset force. The BNF approach is finally applied to the study of the motion of PCs driven by flows through complex geometries (pipes, porous media, ...).
\end{abstract}

